Bull. Austral. Math. Soc.

$13 \mathrm{~F} 10,13 \mathrm{M} 10,13 \mathrm{P} 10$

VOL. 65 (2002) [145-152]

\title{
GRÖBNER BASES AND PRODUCTS OF COEFFICIENT RINGS
}

\author{
Graham H. Norton and ANa SăLĂgean
}

\begin{abstract}
Suppose that $A$ is a finite direct product of commutative rings. We show from first principles that a Gröbner basis for an ideal of $A\left[x_{1}, \ldots, x_{n}\right]$ can be easily obtained by 'joining' Gröbner bases of the projected ideals with coefficients in the factors of $A$ (which can themselves be obtained in parallel). Similarly for strong Gröbner bases. This gives an elementary method of constructing a (strong) Gröbner basis when the Chinese Remainder Theorem applies to the coefficient ring and we know how to compute (strong) Gröbner bases in each factor.
\end{abstract}

\section{INTRODUCTION}

Let $A$ be a commutative ring with $1 \neq 0$. We are interested in obtaining a (strong) Gröbner basis of a non-zero ideal $I$ of $A\left[x_{1}, \ldots, x_{n}\right]$ when $A=A_{1} \times \cdots \times A_{m}$ is a direct product of rings and we know how to obtain (strong) Gröbner bases of the projected ideals $\pi_{i}(I)$ for $i=1, \ldots, m$. We show that this can be done by 'joining' (strong) Gröbner bases for the $\pi_{i}(I)$ of $A_{i}\left[x_{1}, \ldots, x_{n}\right]$. Thus we can compute a (strong) Gröbner basis for $I$ when we know algorithms for computing a (strong) Gröbner basis for $\pi_{i}(I)$. As an application, we compute a (strong) Gröbner basis for $I$ when the Chinese Remainder Theorem applies to $A$ and we can compute (strong) Gröbner bases in each factor. Recall that if $A$ is a principal ideal ring, any non-zero ideal of $A[\mathbf{x}]$ has a strong Gröbner basis [3, Algorithm 6.4]. We give another proof of this fact.

The preliminary Section 2 recalls the necessary background on (strong) Gröbner bases from $[1,3]$. Section 3 discusses the join of Gröbner bases while Section 4 describes the strong join of strong Gröbner bases. In the final section, we assume that $A$ is a principal ideal ring.

Received 9th August, 2001.

Research supported by the United Kingdom Engineering and Physical Sciences Research Council under Grant L07680 in the Algebraic Coding Research Group. Centre for Communications Research, University of Bristol, U.K.

Copyright Clearance Centre, Inc. Serial-fee code: 0004-9727/02 \$A2.00+0.00. 


\section{Preliminaries}

We have $A=A_{1} \times \cdots \times A_{m}$ and we write $A[\mathbf{x}]$ for $A\left[\dot{x}_{1}, \ldots, x_{n}\right]$. The monoid of terms in $x_{1}, \ldots, x_{n}$ is denoted by $T$. Let $<$ be a fixed but arbitrary admissible order on $T$. Throughout the paper, we use the same term order $<$ on each $A_{i}[\mathbf{x}]$ as on $A[\mathbf{x}]$.

If $f=\sum_{t \in T} f_{t} t \in A[\mathbf{x}] \backslash\{0\}$ and $v=\max \left\{t \in T: f_{t} \neq 0\right\}$ then $v$ is the leading term, $f_{v}$ the leading coefficient and $f_{v} v$ the leading monomial of $f$, denoted $\operatorname{lt}(f), \operatorname{lc}(f)$ and $\operatorname{lm}(f)$ respectively. We also write $\operatorname{lm}(S)$ for $\{\operatorname{lm}(f): f \in S\}$ where $S \subset A[\mathbf{x}] \backslash\{0\}$.

Let $G \subset A[\mathbf{x}] \backslash\{0\}$ be finite. Then $f \in A[\mathbf{x}]$ has a standard representation with respect to $G$ if $f=\sum_{j=1}^{k} c^{(j)} t^{(j)} g^{(j)}$ for some $c^{(j)} \in A \backslash\{0\}, t^{(j)} \in T, g^{(j)} \in G$ such that $t^{(j)} \operatorname{lt}\left(g^{(j)}\right) \leqslant \operatorname{lt}(f), \quad[2$, p. 218]. We write $\operatorname{Std}(G)$ for the polynomials which have a standard representation with respect to $G$.

Also, if $G \subset A[\mathbf{x}] \backslash\{0\}$ is finite, then $G$ is a Gröbner basis for a non-zero ideal $I \subset A[\mathbf{x}]$ if and only if $I=\operatorname{Std}(G),[1$, Theorem 4.1.12]. If $A$ is Noetherian, every non-zero ideal of $A[\mathbf{x}]$ has a Gröbner basis [1, Corollary 4.1.17].

Recall that if $G \subset A[\mathbf{x}] \backslash\{0\}$ is finite, then $G$ is a strong Gröbner basis for $I=\langle G\rangle$ if and only if for any $f \in I$ there is a $g \in G$ such that $\operatorname{lm}(g) \mid \operatorname{lm}(f),[\mathbf{1}$, Definition 4.5.6]. If $A$ is a principal ideal ring, Algorithm 6.4 of [3] constructs a strong Gröbner basis for any non-zero ideal of $A[\mathbf{x}]$. Also, a strong Gröbner basis $G$ is called minimal if no proper subset of $G$ is a strong Gröbner basis for $\langle G\rangle$.

\section{THE JOIN}

The projections $\pi_{i}: A \rightarrow A_{i}$ induce maps $\pi_{i}: A[\mathbf{x}] \rightarrow A_{i}[\mathbf{x}]$. It is straightforward to check that the induced map $\pi: A[\mathbf{x}] \rightarrow A_{1}[\mathbf{x}] \times \cdots \times A_{m}[\mathbf{x}]$ given by $\pi(f)=\left(\pi_{1}(f), \ldots, \pi_{m}(f)\right)$ and the map $\kappa: A_{1}[\mathbf{x}] \times \cdots \times A_{m}[\mathbf{x}] \rightarrow A[\mathbf{x}]$, which collects coefficients of like terms, are mutually inverse ring homomorphisms. We relate Gröbner bases of $I \subset A[\mathbf{x}]$ to Gröbner bases of $\pi_{i}(I) \subset A_{i}[\mathbf{x}]$, where $1 \leqslant j \leqslant m$.

PRopos ITION 3.1. If $G$ is a Gröbner basis for a non-zero ideal $I \subset A[\mathbf{x}]$, then $\pi_{i}(G) \backslash\{0\}$ is a Gröbner basis for $\pi_{i}(I)$ in $A_{i}[\mathbf{x}]$ for $i=1, \ldots, m$.

ProOF: We can assume that $i=1$. Let $f_{1} \in \pi_{1}(I) \backslash\{0\} \subset A_{1}[\mathbf{x}]$ and put $G_{1}=$ $\pi_{1}(G) \backslash\{0\}$. We show that $f_{1} \in \operatorname{Std}\left(G_{1}\right)$. For let $f=\kappa\left(f_{1}, 0, \ldots, 0\right) \in I \backslash\{0\}$. We have $\operatorname{lm}(f)=\left(\operatorname{lc}\left(f_{1}\right), 0, \ldots, 0\right) \operatorname{lt}\left(f_{1}\right)$, so that $\operatorname{lt}(f)=\operatorname{lt}\left(f_{1}\right)$. Since $G$ is a Gröbner basis for $I$, $f=\sum_{j=1}^{k} c^{(j)} t^{(j)} g^{(j)}$ for some $c^{(j)} \in A \backslash\{0\}, t^{(j)} \in T, g^{(j)} \in G$ with $t^{(j)} \operatorname{lt}\left(g^{(j)}\right) \leqslant \operatorname{lt}(f)=$ $\operatorname{lt}\left(f_{1}\right)$. Then $f_{1}=\sum_{r=1}^{s} \pi_{1}\left(c^{\left(j_{r}\right)}\right) t^{\left(j_{r}\right)} \pi_{1}\left(g^{\left(j_{r}\right)}\right)$ for some $j_{r}, 1 \leqslant j_{1}<\cdots<j_{s} \leqslant k$ with all $\pi_{1}\left(c^{\left(j_{r}\right)}\right)$ and $\pi_{1}\left(g^{\left(j_{r}\right)}\right)$ non-zero. We have $t^{\left(j_{r}\right)} \operatorname{lt}\left(\pi_{1}\left(g^{\left(j_{r}\right)}\right)\right) \leqslant t^{\left(j_{r}\right)} \operatorname{lt}\left(g^{\left(j_{r}\right)}\right) \leqslant \operatorname{lt}(f)=\operatorname{lt}\left(f_{1}\right)$, that is, $f_{1} \in \operatorname{Std}\left(G_{1}\right)$ and $G_{1}$ is a Gröbner basis for $\pi_{1}(I)$. 
Definition 3.2: Let $G_{i} \subset A_{i}[\mathbf{x}] \backslash\{0\}$ for $i=1,2$. Then, $G_{1} \cup G_{2}$, the join of $G_{1}$ and $G_{2}$ is the subset $G_{1} \times\{0\} \cup\{0\} \times G_{2}$ of $A_{1}[\mathrm{x}] \times A_{2}[\mathbf{x}]$.

Proposition 3.3. Let $I$ be a non-zero ideal of $A[\mathbf{x}]$ and $G_{i} \subset A_{i}[\mathbf{x}] \backslash\{0\}$ for $i=1, \ldots, m$. Then $\kappa\left(G_{1} \sqcup \cdots \sqcup G_{m}\right)$ is a Gröbner basis for $I$ if and only if $G_{i}$ is a Gröbner basis for $\pi_{i}(I)$ for $i=1, \ldots, m$.

Proof: Note first that $0 \notin H=\kappa\left(G_{1} \cup \cdots \sqcup G_{m}\right)$. We show $I \subset \operatorname{Std}(H)$ if each $G_{i}$ is a Gröbner basis. Let $f \in I \backslash\{0\}$. Since $\pi_{i}(f) \in \pi_{i}(I)=\operatorname{Std}\left(G_{i}\right)$, we can write $\pi_{i}(f)=\sum_{j=1}^{k_{i}} c_{i}^{(j)} t_{i}^{(j)} g_{i}^{(j)}$ for some $k_{i} \geqslant 1, c_{i}^{(j)} \in A_{i} \backslash\{0\}, t_{i}^{(j)} \in T, g_{i}^{(j)} \in G_{i}$ with $t_{i}^{(j)} \operatorname{lt}\left(g_{i}^{(j)}\right) \leqslant \operatorname{lt}\left(\pi_{i}(f)\right) \leqslant \operatorname{lt}(f)$. Then

$$
\begin{aligned}
f & =\kappa\left(\pi_{1}(f), \ldots, \pi_{m}(f)\right) \\
& \left.=\kappa\left(\sum_{j=1}^{k_{1}} c_{1}^{(j)} t_{1}^{(j)} g_{1}^{(j)}, 0, \ldots, 0\right)\right)+\cdots+\kappa\left(0, \ldots, 0, \sum_{j=1}^{k_{m}} c_{m}^{(j)} t_{m}^{(j)} g_{m}^{(j)}\right) \\
& =\sum_{j=1}^{k_{1}} c_{1}^{(j)} t_{1}^{(j)} \kappa\left(g_{1}^{(j)}, 0, \ldots, 0\right)+\cdots+\sum_{j=1}^{k_{m}} c_{m}^{(j)} t_{m}^{(j)} \kappa\left(0, \ldots, 0, g_{m}^{(j)}\right)
\end{aligned}
$$

Now $\kappa\left(0, \ldots, 0, g_{i}^{(j)}, 0, \ldots, 0\right) \in H, t_{i}^{(j)} \operatorname{lt}\left(\kappa\left(0, \ldots, 0, g_{i}^{(j)}, 0, \ldots, 0\right)\right)=t_{i}^{(j)} \operatorname{lt}\left(g_{i}^{(j)}\right)$ $\leqslant \operatorname{lt}(f)$ for $j=1, \ldots, k_{i}$ and $i=1, \ldots, m$, so that $f \in \operatorname{Std}(H)$. The converse follows immediately from Proposition 3.1 .

ExAmple 3.4. Let $f=2 x^{2}+3 x+1 \in \mathbb{Z}_{6}[x]$. We obtain a Gröbner basis for $\langle f\rangle$ as follows. The usual isomorphism $\chi: \mathbb{Z}_{6} \rightarrow \mathbb{Z}_{2} \times \mathbb{Z}_{3}$ induces an isomorphism $\chi: \mathbb{Z}_{6}[x] \rightarrow$ $\left(\mathbb{Z}_{2} \times \mathbb{Z}_{3}\right)[x]$ and $\chi(f)=(0,2) x^{2}+(1,0) x+(1,1)$. We have $\pi \chi(f)=\left(x+1,2 x^{2}+1\right)$ $\in \mathbb{Z}_{2}[x] \times \mathbb{Z}_{3}[x]$ and clearly $\{x+1\}$ and $\left\{x^{2}+2\right\}$ are Gröbner bases in $\mathbb{Z}_{2}[x]$ and $\mathbb{Z}_{3}[x]$ respectively. By Proposition 3.3, $\kappa\left(\{x+1\} \sqcup\left\{x^{2}+2\right\}\right)=\left\{(1,0) x+(1,0),(0,1) x^{2}+(0,2)\right\}$ is a Gröbner basis for $\langle\chi(f)\rangle$ and we deduce that $\chi^{-1} \kappa\left(\{x+1\} \sqcup\left\{x^{2}+2\right\}\right)=\{3(x+$ $\left.1), 4 x^{2}+2\right\}$ is a Gröbner basis for $\langle f\rangle$.

\section{THE STRONG JOIN}

First note that $G=\left\{3(x+1), 4 x^{2}+2\right\}$ is not a strong Gröbner basis for $\langle G\rangle$ in Example 3.4: $x^{2}-3 x+2=4 x^{2}+2-3 x(x+1) \in\langle G\rangle$, but 3 and 4 are not units in $\mathbb{Z}_{6}$, so there is no $g \in G$ such that $\operatorname{lm}(g) \mid \operatorname{lm}\left(x^{2}-3 x+2\right)$. We shall now show how to obtain a strong Gröbner basis in $A[\mathbf{x}]$ from strong Gröbner bases in the $A_{i}[\mathbf{x}]$.

PRopos ITION 4.1. If $G$ is a strong Gröbner basis for a non-zero ideal $I \subset A[\mathbf{x}]$ then $\pi_{i}(G) \backslash\{0\}$ is a strong Gröbner basis for $\pi_{i}(I)$ in $A_{i}[\mathbf{x}]$ for $i=1, \ldots, m$.

Proof: We take $i=1$. Let $G$ be a strong Gröbner basis and let $f_{1} \in \pi_{1}(I) \backslash\{0\} \subset$ $A_{1}[\mathbf{x}]$. Put $f=\kappa\left(f_{1}, 0, \ldots, 0\right)$ as in Proposition 3.1. There is a $g \in G$ such that 
$\operatorname{lm}(g) \mid \operatorname{lm}(f)$, so $\pi_{1}(\operatorname{lm}(g)) \mid \operatorname{lm}\left(f_{1}\right)$. This means that $\pi_{1}(\operatorname{lm}(g)) \neq 0$, so $\pi_{1}(g) \neq 0$ and $\pi_{1}(\operatorname{lm}(g))=\operatorname{lm}\left(\pi_{1}(g)\right)$. Since $\operatorname{lm}\left(\pi_{1}(g)\right) \mid \operatorname{lm}\left(f_{1}\right)$ and $\pi_{1}(g) \in \pi_{1}(G) \backslash\{0\}, \pi_{1}(G) \backslash\{0\}$ is a strong Gröbner basis for $\left\langle\pi_{1}(G)\right\rangle=\pi_{1}(I)$.

Definition 4.2: Let $G_{i} \subset A_{i}[\mathbf{x}] \backslash\{0\}$ for $i=1,2$. Then $G_{1} \sqsubseteq G_{2}$, the strong join of $G_{1}, G_{2}$ is the subset $G_{1} \cup G_{2} \cup\left\{\left(t_{1} g_{1}, t_{2} g_{2}\right): g_{i} \in G_{i}, t_{i}=\operatorname{lcm}\left(\operatorname{lt}\left(g_{1}\right), \operatorname{lt}\left(g_{2}\right)\right) / \operatorname{lt}\left(g_{i}\right)\right\}$ of $A_{1}[\mathbf{x}] \times A_{2}[\mathbf{x}]$.

PROPOSITION 4.3. $\quad \kappa\left(\kappa\left(G_{1} \unrhd G_{2}\right) \bigsqcup G_{3}\right)=\kappa\left(G_{1} \bigsqcup \kappa\left(G_{2} \sqsubseteq G_{3}\right)\right)$.

PROOF: Use the fact that in $\kappa\left(G_{1} \biguplus G_{2}\right), \operatorname{lt}\left(\kappa\left(t_{1} g_{1}, t_{2} g_{2}\right)\right)=\operatorname{lcm}\left(\operatorname{lt}\left(g_{1}\right), \operatorname{lt}\left(g_{2}\right)\right)$ and that the $\mathrm{lcm}$ of leading terms is associative.

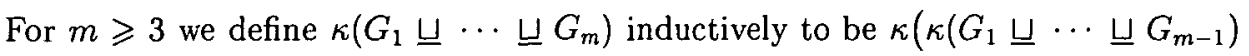
$\left.\bigsqcup G_{m}\right)$.

Theorem 4.4. Let $I$ be a non-zero ideal in $A[\mathbf{x}]$ and $G_{i} \subseteq \pi_{i}(I) \backslash\{0\}$ for $i=1, \ldots, m$. Then $\kappa\left(G_{1} џ \cdots џ G_{m}\right)$ is a strong Gröbner basis for $I$ if and only if $G_{i}$ is a strong Gröbner basis for $\pi_{i}(I)$ for $i=1, \ldots, m$.

Proof: It suffices to prove the result for $m=2$, as the general case follows inductively. Assume that $G_{i}$ is a strong Gröbner basis for $\pi_{i}(I)$ for $i=1,2$. We shall prove that for any $f \in I \backslash\{0\}$ there is a $g \in \kappa\left(G_{1} \sqsubseteq G_{2}\right)$ such that $\operatorname{lm}(g) \mid \operatorname{lm}(f)$. For $i=1,2$, put $\pi_{i}(f)=f_{i}$. We consider several cases.

(i) $f_{1} \neq 0$ and $f_{2}=0$. Then $\operatorname{lm}(f)=\left(\operatorname{lc}\left(f_{1}\right), 0\right) \operatorname{lt}\left(f_{1}\right)$. Since $G_{1}$ is a strong Gröbner basis for $\pi_{1}(I)$, there is a $g_{1} \in G_{1}$ such that $\operatorname{lm}\left(g_{1}\right) \mid \operatorname{lm}\left(f_{1}\right)$. Putting $g=\kappa\left(g_{1}, 0\right) \in \kappa\left(G_{1}\left\lfloor G_{2}\right)\right.$, we have $\operatorname{lm}(g)=\left(\operatorname{lc}\left(g_{1}\right), 0\right) \operatorname{lt}\left(g_{1}\right)$ and so $\operatorname{lm}(g) \mid \operatorname{lm}(f)$.

(ii) $f_{1} \neq 0, f_{2} \neq 0$ and $\operatorname{lt}\left(f_{1}\right)>\operatorname{lt}\left(f_{2}\right)$ : this is similar to case (i) since $\operatorname{lm}(f)=$ $\left(\operatorname{lc}\left(f_{1}\right), 0\right) \operatorname{lt}\left(f_{1}\right)$.

(iii) $f_{1}=0$ and $f_{2} \neq 0$ : this is analogous to case (i).

(iv) $f_{1} \neq 0, f_{2} \neq 0$ and $\operatorname{lt}\left(f_{1}\right)<\operatorname{lt}\left(f_{2}\right)$ : see case (iii).

(v) $f_{1} \neq 0, f_{2} \neq 0$ and $\operatorname{lt}\left(f_{1}\right)=\operatorname{lt}\left(f_{2}\right)$. Then $\operatorname{lm}(f)=\left(\operatorname{lc}\left(f_{1}\right), \operatorname{lc}\left(f_{2}\right)\right) \operatorname{lt}\left(f_{1}\right)$. For $i=1,2$, let $g_{i} \in G_{i}$ be such that $\operatorname{lm}\left(g_{i}\right) \mid \operatorname{lm}\left(f_{i}\right)$. Putting $g=$ $\kappa\left(t_{1} g_{1}, t_{2} g_{2}\right) \in \kappa\left(G_{1} \sqsupseteq G_{2}\right)$, where $t_{i}$ is as in Definition 4.2 , we have $\operatorname{lm}(g)=\left(\operatorname{lc}\left(g_{1}\right), \operatorname{lc}\left(g_{2}\right)\right) \operatorname{lcm}\left(\operatorname{lt}\left(g_{1}\right), \operatorname{lt}\left(g_{2}\right)\right)$ and so $\operatorname{lm}(g) \mid \operatorname{lm}(f)$.

For the converse, assume that $\kappa\left(G_{1} \unrhd G_{2}\right)$ is a strong Gröbner basis for $I$ and fix $i \in\{0,1\}$. Let $H_{i}=\pi_{i}\left(G_{1} \succeq G_{2}\right) \backslash\{0\}$, which is a strong Gröbner basis for $\pi_{i}(I)$ by Proposition 4.1. From the definition of $G_{1} џ G_{2}, G_{i} \subseteq H_{i}$ and any $h_{i} \in H_{i} \backslash G_{i}$ is of the form $h_{i}=t_{i} g_{i}$ for some $t_{i} \in T$. Thus $\left\langle G_{i}\right\rangle=\left\langle H_{i}\right\rangle$ and for any $f \in \pi_{i}(I)=\left\langle G_{i}\right\rangle$, there is an $h_{i} \in H_{i}$ and a $g_{i} \in G_{i}$ such that $\operatorname{lm}\left(g_{i}\right)\left|\operatorname{lm}\left(h_{i}\right)\right| \operatorname{lm}(f)$. Hence $G_{i}$ is a strong Gröbner basis for $\pi_{i}(I)$.

Theorem 4.4 thus gives an iterative algorithm for computing a strong Gröbner basis in $A[\mathbf{x}]$, provided we have an algorithm ( $\mathrm{SGB}_{\boldsymbol{i}}$ say) that computes a strong Gröbner 
basis in each $A_{i}[\mathrm{x}]$ for $1 \leqslant i \leqslant m$. The $\mathrm{SGB}_{i}$ can be done in parallel and the complexity of computing $\kappa\left(G_{1} \underline{\cup} \cdots \underline{\cup} G_{m}\right)$ from $G_{1}, \ldots, G_{m}$ is $\mathcal{O}\left(\prod_{i=1}^{m}\left|G_{i}\right|\right)$. The latter can be improved by first minimising each $G_{i}$. We note that $\kappa\left(G_{1} \underline{\sqcup} \cdots \underline{G_{m}}\right)$ may not be minimal, so in general, a further minimisation step will be necessary. We formalise this as follows.

\section{Algorithm 4.5.}

Input: $\quad F \subset A[\mathbf{x}] \backslash\{0\}, F$ finite, $A=\prod_{i=1}^{m} A_{i}$ and we have an algorithm strong $\mathrm{SGB}_{i}$ which computes a strong Gröbner basis in $A_{i}[\mathbf{x}]$ for $1 \leqslant i \leqslant m$.

Output: $G$, a minimal strong Gröbner basis for $\langle F\rangle$.

\section{begin}

for $i \leftarrow 1$ to $m$ do

$$
G_{i} \leftarrow \operatorname{SGB}_{i}\left(\pi_{i}(F)\right)
$$

minimise $G_{i}$

\section{end for}

$G \leftarrow G_{1}$

$$
\text { for } i \leftarrow 2 \text { to } m \text { do }
$$

$$
G \leftarrow \kappa\left(G \biguplus G_{i}\right)
$$

end for

minimise $G$

$\operatorname{return}(G)$

end

Finally, we note that in computing $G=\kappa\left(G_{1} \underline{\Perp} \cdots \underline{\cup} G_{m}\right)$ we can first compute $\operatorname{lm}(G)$ to preselect the polynomials of $G$ belonging to a minimal strong Gröbner basis. Only these polynomials need then be computed in full. See Example 5.3.

\section{ThE PRINCIPAL IDEAL RING CASE}

In this final section, we restrict $A$ to be a principal ideal ring. We give an alternative proof that any non-zero ideal of $A[\mathbf{x}]$ has a strong Gröbner basis and conclude with some examples.

Corollary 5.1. (Compare [3, Algorithm 6.4].) If $A$ is a principal ideal ring then any non-zero ideal of $A[\mathbf{x}]$ has a strong Gröbner basis.

Proof: We have $A \cong \prod_{i=1}^{m} A_{i}$, where each $A_{i}$ is a principal ideal domain or a finitechain ring by [4, Theorem 33, Section 15 , Chapter 4]. We can obtain a strong Gröbner basis over a principal ideal domain using for example, [2, Algorithm D-Gröbner, p. 461]). Over a finite-chain ring any Gröbner basis is a strong Gröbner basis by [3, Proposition 
3.9], so it suffices to compute a Gröbner basis, using for example [3, Algorithm 6.1] which computes a Gröbner basis over any principal ideal ring. Hence by Theorem 4.4 we can compute a strong Gröbner basis for any non-zero ideal of $A[\mathbf{x}]$.

An improved strong Gröbner basis algorithm for finite-chain rings is described in the Appendix.

Example 5.2. (Compare [3, Example 7.3].) Let $F=\left\{2 x^{2}+3 x+1\right\} \subset \mathbb{Z}_{6}[x]$, as in Example 3.4. We obtain a strong Gröbner basis for $\langle F\rangle$ by applying Algorithm 4.5 to $\chi(F)$. Firstly, $\pi \chi(F)=\left(x+1,2 x^{2}+1\right)$ and trivially $\{x+1\}$ and $\left\{x^{2}+2\right\}$ are minimal strong Gröbner bases in $\mathbb{Z}_{2}[x]$ and $\mathbb{Z}_{3}[x]$ respectively. We have $\{x+1\} \bigsqcup\left\{x^{2}+2\right\}=$ $\left\{(x+1,0),\left(0, x^{2}+2\right),\left(x^{2}+x, x^{2}+2\right)\right\}$ and $G=\kappa\left(\{x+1\} \leqq\left\{x^{2}+2\right\}\right)=\{(1,0) x+$ $\left.(1,0),(0,1) x^{2}+(0,2),(1,1) x^{2}+(1,0) x+(0,2)\right\}$ is a strong Gröbner basis for $\langle\chi(F)\rangle$. We minimise $G$ to obtain $H=\left\{(1,0) x+(1,0),(1,1) x^{2}+(1,0) x+(0,2)\right\}$. Finally $\chi^{-1}(H)=\left\{x^{2}+3 x+2,3(x+1)\right\}$ is a minimal strong Gröbner basis for $\langle F\rangle$.

In the next example, we use Algorithm SGB-FCR of the Appendix.

EXAmple 5.3. As in [1, Example 4.2.12], let $F=\left\{4 x y+x, 3 x^{2}+y\right\} \subset \mathbb{Z}_{20}[x, y]$. Using lexicographic order with $x>y$, they obtain a Gröbner basis $G^{\prime}=\left\{3 x^{2}+y, 4 x y+\right.$ $\left.x, 5 x, 4 y^{2}+y, 15 y\right\}$ via the method of syzygy modules. This is not a strong Gröbner basis since $x y-x=5 x y-(4 x y+x)$ is not strongly reducible with respect to $G^{\prime}$. Likewise for $y^{2}-y=5 y^{2}-\left(4 y^{2}+y\right)$. (We note that [3, Corollary 5.12] shows that $\left\{x^{2}+7 y, x y-x, 5 x, y^{2}-y, 5 y\right\}$ is a minimal strong Gröbner basis.)

Instead, we compute a strong Gröbner basis for $\langle F\rangle$ from scratch using the usual isomorphism $\chi: \mathbb{Z}_{20} \rightarrow \mathbb{Z}_{4} \times \mathbb{Z}_{5}$ and Algorithm 4.5. We have $\pi \chi(F)=\{(x, 4 x y+$ $\left.x),\left(3 x^{2}+y, 3 x^{2}+y\right)\right\} \subset \mathbb{Z}_{4}[x] \times \mathbb{Z}_{5}[x]$.

We obtain $G_{1}=\{x, y\}$ as a strong Gröbner basis for $\left\{x, 3 x^{2}+y\right\}$ using Algorithm SGB-FCR; alternatively $G_{1}$ is a Gröbner basis by [3, Theorem 4.10] and it is a (minimal) strong Gröbner basis by [3, Proposition 3.9]. In $\mathbb{Z}_{5}[x, y]$, we work with $\left\{x y+4 x, x^{2}+2 y\right\}$. A minimal strong Gröbner basis is $G_{2}=\left\{x y+4 x, x^{2}+2 y, y^{2}+4 y\right\}$. First computing $\operatorname{lm}\left(\kappa\left(G_{1} \underline{\cup} G_{2}\right)\right)$ yields $H=\left\{(1,1) x^{2}+(0,2) y,(1,1) x y+(0,4) x,(1,0) x,(1,1) y^{2}+\right.$ $(0,4) y,(1,0) y\}$ as a minimal strong Gröbner basis for $\langle\chi(F)\rangle$. So $\chi^{-1}(H)=\left\{x^{2}+\right.$ $\left.12 y, x y+4 x, 5 x, y^{2}+4 y, 5 y\right\}$ is a minimal strong Gröbner basis for $\langle F\rangle$.

\section{APPENDIX}

We derive an algorithm for computing a strong Gröbner basis over a finite-chain ring $R$ from [3, Algorithm 6.1], using the definitions and notation of [3, Sections 3.1, 4.3]. In particular, for $f, f_{1}, f_{2} \in R[\mathbf{x}] \backslash\{0\}$ and a finite set $G$ of non-zero polynomials, $\operatorname{Spol}\left(f_{1}, f_{2}\right), \operatorname{Apol}(f), \operatorname{Rem}(f, G), \operatorname{SRem}(f, G)$ denote the set of S-polynomials of $f_{1}, f_{2}$, the set of A-polynomials of $f$, the remainder and the strong remainder of $f$ with respect to $G$, respectively. 
Algorithm 6.1 of [3] computes a Gröbner basis over any principal ideal ring, so in particular over $R$. We know that any Gröbner basis over $R$ is a strong Gröbner basis by [3, Proposition 3.9]. We also know that $f$ is reducible with respect to $G$ if and only if $f$ is strongly reducible with respect to $G$ by $[3$, Proposition 3.2], so that $\operatorname{SRem}(f, G) \subseteq \operatorname{Rem}(f, G)$. So over $R$ we only need to use strong reduction, which is more efficient than reduction. The improved algorithm follows.

ALGORITHM 6.1.

$G \leftarrow \operatorname{SGB-FCR}(F)$

Input: $\quad F$ a finite subset of $R[\mathbf{x}] \backslash\{0\}$, where $R$ is a computable finite-chain ring.

Output: $G$ a strong Gröbner basis for $\langle F\rangle$.

Notes: $B$ is the set of pairs of polynomials in $G$ whose S-polynomials still have to be computed.

$C$ is the set of polynomials in $G$ whose A-polynomials still have to be computed.

begin

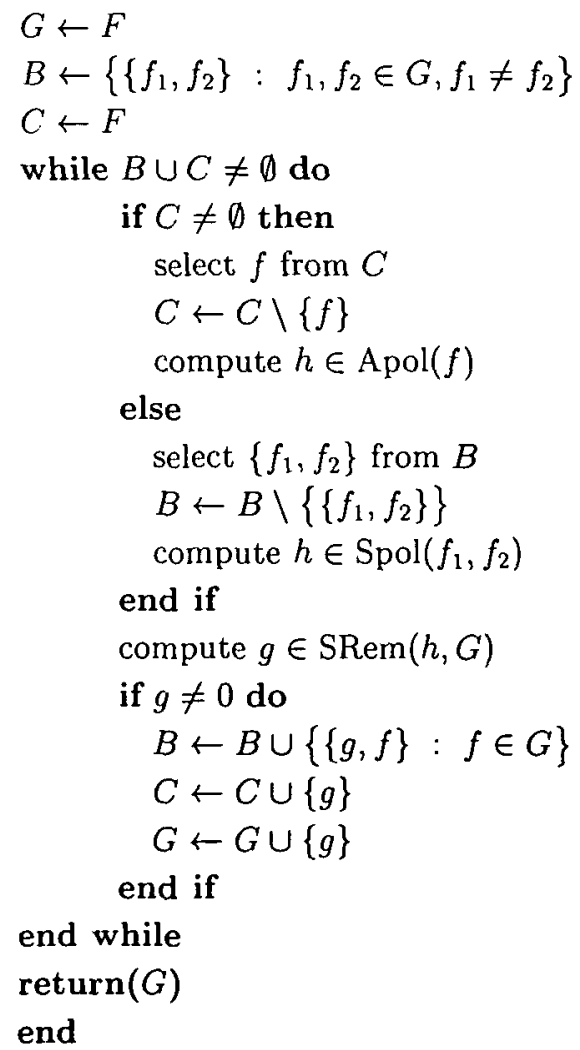




\section{REFERENCES}

[1] W. Adams and P. Loustaunau, An introduction to Gröbner bases, Graduate Studies in Mathematics 3 (American Mathematical Society, Providence, R.I., 1994.).

[2] T. Becker and V. Weispfenning, Gröbner bases, Graduate Texts in Mathematics 141 (Springer-Verlag, New York, 1993).

[3] G.H. Norton and A. Sălăgean, 'Strong Gröbner bases for polynomials over a principal ideal ring', Bull. Austral. Math. Soc. 64 (2001), 505-528.

[4] O. Zariski and P. Samuel, Commutative algebra, Graduate Texts in Mathematics 29, Volume 1 (Springer-Verlag, Heidelberg, New York, 1979).

Department of Mathematics

The University of Queensland

Queensland 4072

Australia
Department of Computer Science

Loughborough University

Leicestershire LE11 3TU

United Kingdom 